\title{
Capsaicin-Sensitive Sensory Nerves Indirectly Modulate Motor Function of the Urinary Bladder
}

\author{
Hsi-Hsien Chang ${ }^{1}$, Shang-Jen Chang ${ }^{1,2}$, Cheng-Hsing Hsieh ${ }^{1}$, Chun-Kai Hsu ${ }^{1}$, Stephen Shei-Dei Yang ${ }^{1,2,3}$ \\ ${ }^{1}$ Division of Urology, Taipei Tzu Chi Hospital, New Taipei, Taiwan \\ ${ }^{2}$ School of Medicine, Buddhist Tzu Chi University, Hualien, Taiwan \\ ${ }^{3}$ Taipei Tzu Chi Hospital, Buddhist Medical Foundation, New Taipei, Taiwan
}

Purpose: The urinary bladder (UB) is innervated by both sensory and autonomic nerves. Recent studies have shown that sensory neuropeptides induced contractions in the detrusor muscle. Therefore, in a mouse model, we investigated the presence of interactions between the submucosal sensory nerves and the autonomic nerves that regulate the motor function of the detrusor muscle.

Methods: UB samples from male C57BL/6 mice were isolated, cut into strips, and mounted in an organ bath. Dose-response curves to norepinephrine and phenylephrine were studied in UB strips with and without mucosa, and the effects of preincubation with a receptor antagonist and various drugs on relaxation were also studied using tissue bath myography.

Results: Phenylephrine-induced relaxation of the UB strips showed concentration-related effects. This relaxation appeared in both mucosa-intact and mucosa-denuded UB strips, and was significantly inhibited by lidocaine, silodosin, and guanethidine (an adrenergic neuronal blocker). Meanwhile, phenylephrine-induced relaxation was inhibited by pretreatment with propranolol and calcitonin gene-related peptide (CGRP)-depletory capsaicin in UB strips with and without mucosa.

Conclusions: The present study suggests that phenylephrine activates the $\alpha-1 \mathrm{~A}$ adrenergic receptor (AR) of the sensory nerve, and then activates capsaicin-sensitive sensory nerves to release an unknown substance that facilitates the release of norepinephrine from adrenergic nerves. Subsequently, norepinephrine stimulates $\beta$-ARs in the detrusor muscle in mice, leading to neurogenic relaxation of the UB. Further animal and human studies are required to prove this concept and to validate its clinical usefulness.

Keywords: Submucosal sensory nerve; Alpha-1 adrenergic receptors; Silodosin; Phenylephrine; Capsaicin

- Research Ethics: All animal experimental procedures were approved by the Institutional Animal Care and Use Committee (IACUC) of Taipei Tzu Chi Hospital (ID \# 105-IACUC-015; 106-IACUC-003), and performed in accordance with the guidelines of the National Institutes of

Health on the care and use of laboratory animals.

- Conflict of Interest: No potential conflict of interest relevant to this article was reported.

\footnotetext{
- HIGHLIGHTS

- Present study demonstrated that the activation of CGRPergic sensory nerve may enhance the release of norepinephrine from adrenergic nerve, and then norepinephrine directly activated the $\beta$-adrenoceptors of detrusor muscle.

- Submucosal sensory nerve may release some unknown neurotransmitter to regulate the motor function of detrusor muscle via adrenergic nerve.
}

Corresponding author: Stephen Shei-Dei Yang (iD https://orcid.org/0000-0003-3814-6980 Division of Urology, Taipei Tzu Chi Hospital, No. 289, Jianguo Rd., Sindian Dist., New Taipei, Taiwan

E-mail: urolyang@tzuchi.com.tw / Tel: +886-66289779 (ext. 5708) / Fax: +886-66289009

Submitted: March 26, 2018 / Accepted after revision: June 7, 2018 


\section{INTRODUCTION}

A previous study reported that phenylephrine induced relaxation in the detrusor muscle in the human bladder, and that this effect was eliminated by propranolol [1]. Phenylephrine is a selective $\alpha-1$ adrenergic receptor (AR) agonist that does not exert a direct effect on $\beta$-ARs [2-5]. Recent studies have shown that tachykinins, a family of neuropeptides released from capsaicin-sensitive afferent nerves when the bladder is distended, modulated vesical tone by acting through tachykinin NK2 receptors [6,7]. The sensory neuropeptides neurokinin A and calcitonin gene-related peptide (CGRP) have been found to be coreleased by sensory fibers after stimulation by either singlepulse electrical field stimulation or capsaicin, which induced contraction of the urinary bladder (UB) [8]. In addition, $\alpha-1$ ARs are functionally expressed by capsaicin-sensitive primary sensory neurons of the urinary tract [9]. During bladder filling, $\alpha-1$ ARs are involved in the activation of the bladder mechanosensory $A \delta$ fibers, which have been found to be inhibited by silodosin (a selective $\alpha-1$ AR antagonists), and $C$ fibers, which have been found to be inhibited by higher doses of silodosin [10]. These results suggest that the activation of $\alpha-1$ ARs in sensory nerves in the UB may cause the release of some neuropeptides that modulate detrusor muscle tone.

Therefore, we conducted this study to examine whether neurogenic relaxation via submucosal sensory neurons takes place in the UB, and if so, to characterize the role of $\alpha-1$ ARs in this interaction.

\section{MATERIALS AND METHODS}

\section{Tissue Preparation}

All animal experimental procedures were approved by the Institutional Animal Care and Use Committee (IACUC) of Taipei Tzu Chi Hospital (ID \# 105-IACUC-015; 106-IACUC-003), and performed in accordance with the guidelines of the $\mathrm{Na}$ tional Institutes of Health on the care and use of laboratory animals. Animals were maintained under controlled light (12-hour light/dark cycles from 7:00 AM to 7:00 PM) and temperature $\left(21^{\circ} \mathrm{C}\right.$ to $\left.23^{\circ} \mathrm{C}\right)$ conditions. Male $\mathrm{C} 57 \mathrm{BL} / 6$ mice were used in all experiments. The male 12-month-old C57BL/6 mice were sacrificed by cervical dislocation after anesthesia with urethane (500 $\mathrm{mg} / \mathrm{kg}$, intraperitoneally) and chloralose $(50 \mathrm{mg} / \mathrm{kg}$, intraperitoneally). The UB was dissected and placed in oxygenated (95\% $\mathrm{O}_{2}$ and $\left.5 \% \mathrm{CO}_{2}\right)$ Krebs solution at $4{ }^{\circ} \mathrm{C}$. The composition of the
Krebs bicarbonate solution (in $\mathrm{mM}$ ) was $\mathrm{NaCl} 117, \mathrm{NaHCO}_{3}$ 25, $\mathrm{KCl} 4.7, \mathrm{CaCl}_{2} 2.5, \mathrm{MgSO}_{4} 1.2, \mathrm{KH}_{2} \mathrm{PO}_{4}$ 1.2, glucose 11.1, and calcium disodium ethylenediamine tetraacetate (EDTA) 0.023 .

\section{Tissue-Bath Wire Myography for the Urinary Bladder}

The UBs were dissected and cleaned of surrounding tissue under a dissecting microscope, and then mounted on a stainlesssteel rod and a platinum wire in a tissue bath containing $20 \mathrm{~mL}$ of Krebs solution that was equilibrated with $95 \% \mathrm{O}_{2}$ and 5\% $\mathrm{CO}_{2}$ and maintained at $37^{\circ} \mathrm{C}$. The mucosa was removed by tissue forceps from to form urothelium-denuded bladder strips. Tension changes were measured by an isometric transducer (FT03C; Grass) and recorded on a Powerlab polygraph (LabChart 7, v7.1. ADInstruments Pty Ltd., Castle Hill, Australia). These UB strips were equilibrated in the Krebs solution for 60 minutes and mechanically stretched to a resting tension of 5 $\mathrm{mN}$ Step 1: After equilibration, the resting muscle tone of the UB strips was changed by cumulative applications of phenylephrine (0.001-10 $\mu \mathrm{M})$. Step 2: After washing, the resting muscle tone of the UB strips was changed by cumulative applications of acetylcholine $(0.001-10 \mu \mathrm{M})$. Between steps 1 and 2, the UB strips were washed for 45 minutes with Krebs solution. Step 3: After the washes were completed, capsaicin (a CGRP depletor, $10 \mu \mathrm{M})$, silodosin $(0.1 \mu \mathrm{M})$, lidocaine $(0.1 \mathrm{mM})$, guanethidine $(10 \mu \mathrm{M})$, or propranolol $(10 \mu \mathrm{M})$ was added $15 \mathrm{~min}$ utes before phenylephrine administration $(0.001-10 \mu \mathrm{M})$, after which the relaxation effects were recorded. Step 4: Maximal contraction of the UB strip was induced by $\mathrm{KCl}(70 \mathrm{mM})$. Only 1 isolated UB strip per animal was used in the myography study.

Changes in muscle contraction tone were estimated as percentages of the $\mathrm{KCl}(70 \mathrm{mM}$ )-induced maximum contraction.

\section{Chemical Denervation With Capsaicin and Effects of Phenylephrine on Bladder Strips With or Without Urothelium}

In the denuded UB strips, the mucosa was removed by tissue forceps under a dissecting microscope. To deplete CGRP in CGRP-containing sensory nerves in the UB, after removing the mucosa, bladder strip segments were incubated in Krebs solution $\left(37^{\circ} \mathrm{C}\right.$ ) containing capsaicin (a CGRP depletor, $10 \mu \mathrm{M}$ ) for 20 minutes and then rinsed for 60 minutes in capsaicin-free Krebs solution $[9,11]$. After washout for 60 minutes, relaxation of the bladder strips with or without mucosa in the absence of active muscle tone was induced by cumulative applications of 
phenylephrine $(0.001-10 \mu \mathrm{M})$.

\section{Drugs Used and Statistical Analysis}

The following chemicals were used: $\mathrm{NaCl}, \mathrm{NaHCO}_{3}, \mathrm{KCl}$, $\mathrm{CaCl}_{2}, \mathrm{MgCl}_{2}$, glucose, $\mathrm{NaH}_{2} \mathrm{PO}_{4}$, EDTA, acetylcholine, capsaicin, guanethidine, lidocaine, propranolol, silodosin, phenylephrine, and norepinephrine (all from Sigma-Aldrich, St. Louis, MO, USA). All values are presented as mean \pm standard error of the mean. The paired $t$-test was used to compare differences in the same strip. P-values of $<0.05$ were considered to indicate statistical significance. Analysis of variance followed by post hoc testing (Bonferroni) was used to compare differences between strips. The Bonferroni test sets the cutoff for significance at $\alpha / n$ ( $\alpha=0.05, n=$ number of groups). Thus, P-values of $<0.025$ ( 0.05 of 2 ) and $<0.0167$ ( 0.05 of 3 ) are statistically significant findings in analyses with 2 and 3 pairs of subjects, respectively.

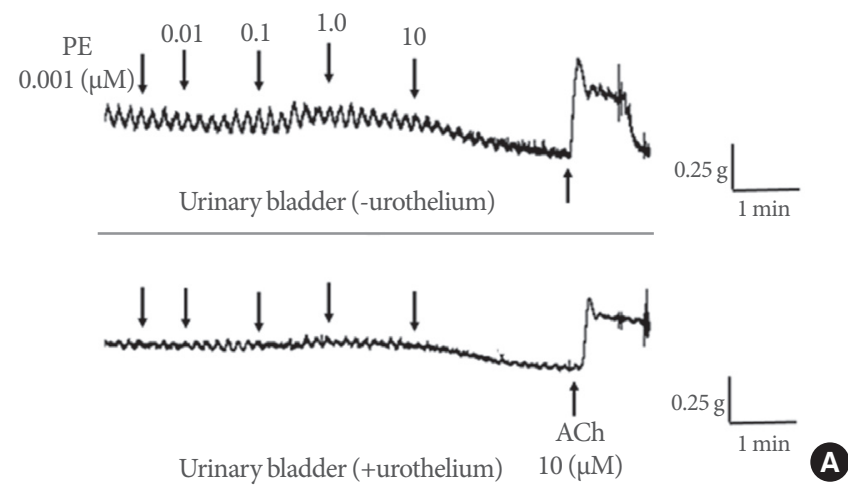

\section{RESULTS}

\section{Effects of Phenylephrine on the Relaxation of Bladder Strips With and Without Urothelium}

A representative tracing in Fig. 1A shows that in the absence of active muscle tone, the bladder strips with $(+)$ or without $(-)$ urothelium relaxed upon application of phenylephrine $(0.001-$ $10 \mu \mathrm{M})$. This phenylephrine-induced relaxation was statistically significantly greater in the urothelium-intact bladder strips than in the urothelium-denuded bladder strips (Fig. 1B, $n=5$; $\mathrm{P}<0.005)$.

\section{Effects of Silodosin, Lidocaine, and Propranolol on Phenylephrine-Induced Relaxation}

In the absence of active muscle tone, the UB strips with mucosa relaxed upon the application of phenylephrine $(0.001-10 \mu \mathrm{M})$ and norepinephrine $(0.001-10 \mu \mathrm{M})$ in a concentration-depen-

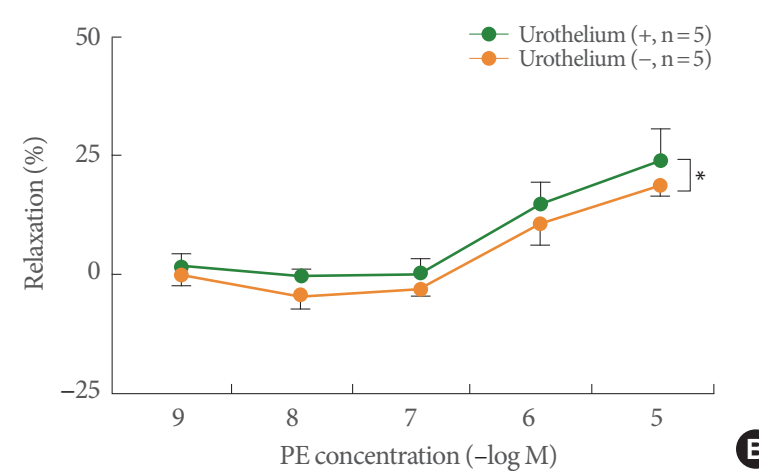

Fig. 1. Phenylephrine (PE)-induced relaxation in urinary bladder strips without (A) upper panel and with urothelium (A) lower panel, showing a concentration-related effect $(\mathrm{PE}, 0.001-10 \mu \mathrm{M})$. The phenylephrine-induced relaxation was significantly greater in urothelium-intact bladder strips than in urothelium-denuded bladder strips (B) $n=5,{ }^{*} \mathrm{P}<0.005$ vs. urothelium [ $]$. Values are mean \pm standard error of the mean. $n$, number of experiments.
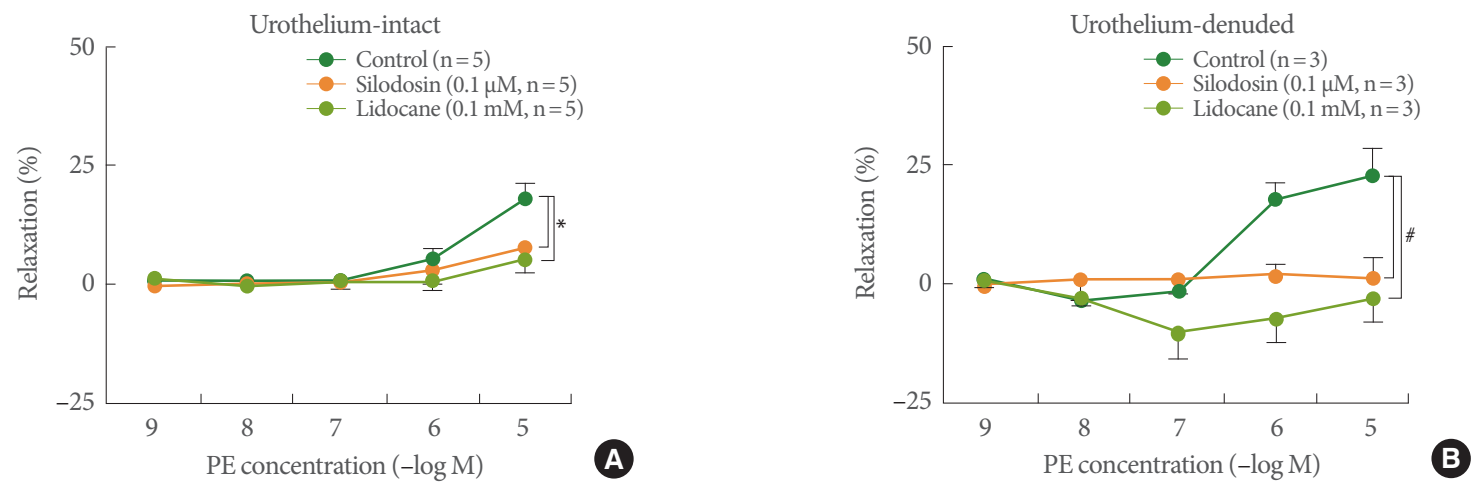

Fig. 2. Phenylephrine (PE)-induced relaxation in urinary bladder strips with (A) and without (B) urothelium was inhibited by silodo$\sin (0.1 \mu \mathrm{M})$, and lidocaine $(0.1 \mathrm{mM}) .{ }^{*} \mathrm{P}<0.005$ vs. control. ${ }^{*} \mathrm{P}<0.005$ vs. control. 

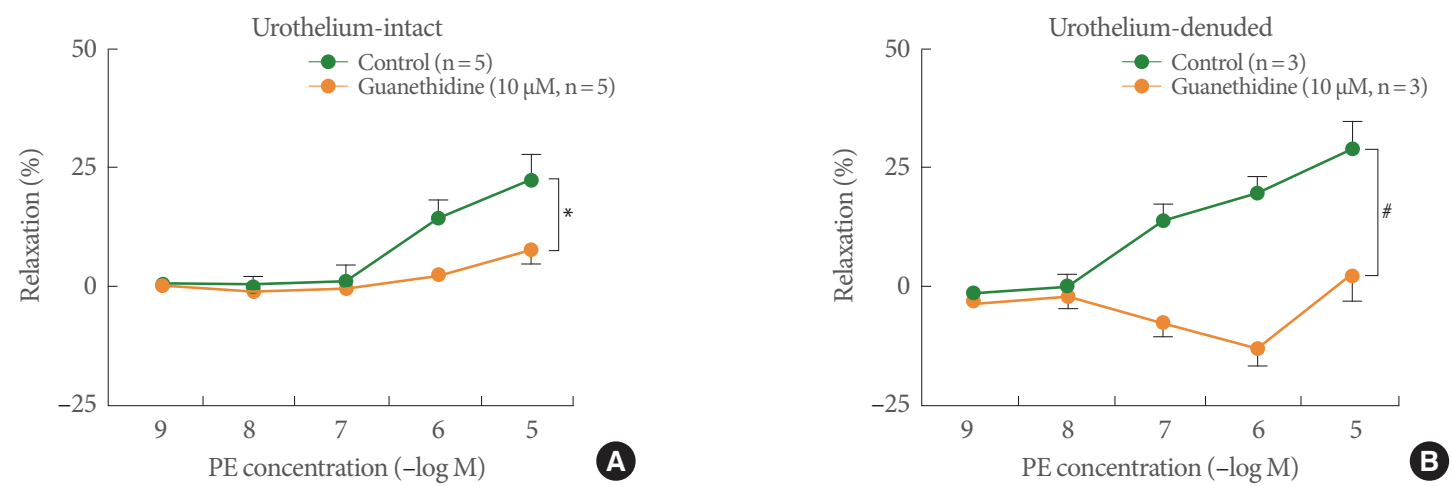

Fig. 3. Phenylephrine (PE)-induced relaxation in urinary bladder strips with (A) and without (B) urothelium was inhibited by guanethidine $(10 \mu \mathrm{M}) .{ }^{*} \mathrm{P}<0.005$ vs. control. ${ }^{*} \mathrm{P}<0.001$ vs. control.
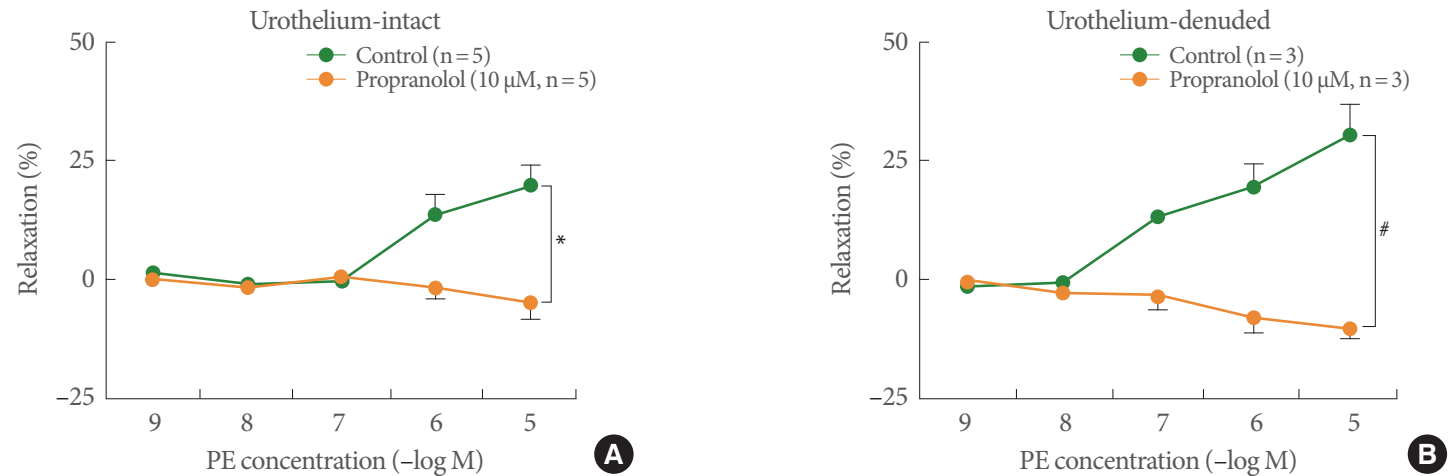

Fig. 4. Phenylephrine (PE)-induced relaxation in urinary bladder strips with (A) and without (B) urothelium was inhibited by propranolol $(10 \mu \mathrm{M})$. Values are mean \pm standard error of the mean. n, number of experiments. ${ }^{*} \mathrm{P}<0.005$ vs. control. ${ }^{*} \mathrm{P}<0.001$ vs. control.
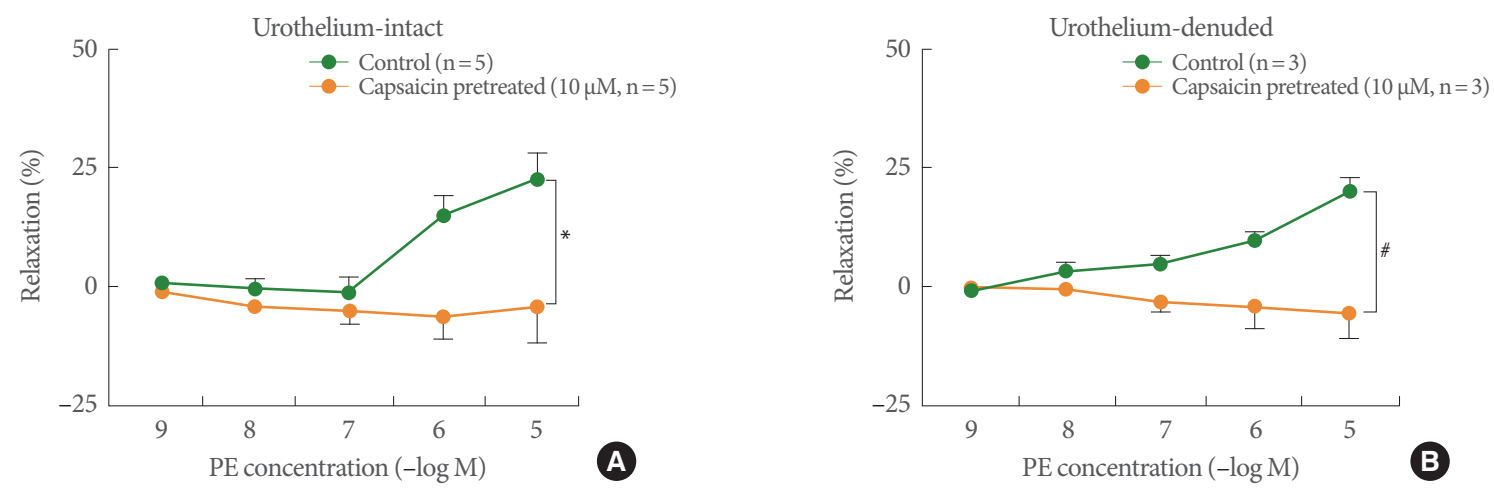

Fig. 5. With pretreatment of capsaicin and washout, phenylephrine (PE) did not relax urinary bladder strips with (A) and without (B) urothelium. Values are mean \pm standard error of the mean. $n$, number of experiments. ${ }^{*} \mathrm{P}<0.001$ vs. control. ${ }^{*} \mathrm{P}<0.001$ vs. control.

dent manner (data not shown). The UB strips with (Fig. 2A, $\mathrm{n}=5$ ) or without (Fig. $2 \mathrm{~B}, \mathrm{n}=3$ ) mucosa relaxed upon application of phenylephrine $(0.001-10 \mu \mathrm{M})$. This phenylephrine-induced relaxation was significantly inhibited by silodosin $(0.1$ $\mu \mathrm{M}, \mathrm{P}<0.005$, Fig. $2 \mathrm{~A}, \mathrm{~B})$ and lidocaine $(0.1 \mathrm{mM}, \mathrm{P}<0.005$,
Fig. 2A, B). Guanethidine $(10 \mu \mathrm{M})$, a known adrenergic neuronal blocker, also inhibited phenylephrine (0.001-10 $\mu \mathrm{M})$ induced relaxation in the UB strips with $(n=5, P<0.005$, Fig. $3 \mathrm{~A}$ ) or without ( $\mathrm{n}=3, \mathrm{P}<0.001$, Fig. $3 \mathrm{~B}$ ) mucosa. Phenylephrine-induced relaxation was likewise significantly impaired by 
propranolol $(10 \mu \mathrm{M})$ in $\mathrm{UB}$ strips with $(\mathrm{n}=5, \mathrm{P}<0.005$, Fig. $4 \mathrm{~A})$ or without $(\mathrm{n}=3, \mathrm{P}<0.001$, Fig. $4 \mathrm{~B})$ mucosa.

\section{Effects of Capsaicin-Mediated Depletion of Neuronal CGRP on Phenylephrine-Induced Relaxation}

After pretreatment and washout of capsaicin, phenylephrine failed to induce concentration-dependent relaxation in UB strips with $(\mathrm{n}=5, \mathrm{P}<0.001$, Fig. $5 \mathrm{~A})$ and without $(\mathrm{n}=3, \mathrm{P}<0.001$, Fig. 5B) mucosa.

\section{DISCUSSION}

Interaction between the submucosal sensory and adrenergic nerves may exist in the UB of mice. Although phenylephrine is a $\alpha-1$ AR agonist, it relaxed strips of detrusor muscle with and without mucosa (Fig. 1). It is well known that the urothelium secretes many signaling molecules that modulate detrusor muscle relaxation and contraction $[12,13]$. Phenylephrine may act on the $\alpha-1$ ARs of urothelium [14,15], and then release some unknown neurotransmitters to facilitate detrusor relaxation. During the process of removing the bladder mucosa, some submucosal nerves may be removed at the same time. The relaxation effect of phenylephrine could thereby be reduced. Both these mechanisms might reduce the ability of phenylephrine to induce relaxation in detrusor muscle without urothelium. However, the role of urothelium in phenylephrineinduced detrusor relaxation seemed to be minor because such effects persisted even after removing the bladder mucosa. The current results suggest that phenylephrine may induce detrusor relaxation via submucosal nerve fibers (Fig. 2).

Phenylephrine-induced detrusor relaxation was found to be related to submucosal CGRPergic nerves, because this relaxation effect was abolished by pretreatment and washout of capsaicin in UB strips with or without mucosa (Fig. 5). The transient receptor potential vanilloid 1 receptor (a capsaicin receptor) has been identified in the urothelium [16]. Capsaicin evoked ATP release from cultured urothelial cells [16] and mucosa-intact UB strips [17], and stimulated afferent nerve activity, which is abolished if capsaicin receptors are absent. After removing the bladder mucosa, phenylephrine still induced relaxation in UB strips, which was also prevented by pretreatment and washout of capsaicin (Fig. 5B). These findings further proved that phenylephrine had a direct effect on CGRPergic nerves, thereby indirectly inducing relaxation in the detrusor muscle.
Phenylephrine-induced relaxation took place via (was associated with) a neurogenic response because both norepinephrine and phenylephrine act on $\beta$-ARs to relax the detrusor muscle. As expected, lidocaine blocked the relaxation effects of phenylephrine (Fig. 2) because it is a sodium channel blocker that inhibits both afferent and efferent nerve activities [18]. This suggests that neurogenic activity may play a role in modulating phenylephrine-induced relaxation. The fact that phenylephrine-induced bladder relaxation was inhibited by guanethidine (Fig. 3) suggests that the relaxation effect of phenylephrine was exerted through enhancement of adrenergic nerve activity. Phenylephrine can be concluded to have induced detrusor relaxation via $\beta$-ARs because the relaxation effect was abolished by propranolol, a nonselective $\beta$-AR blocker (Fig. 4). In the current study, phenylephrine did not induce contraction in UB strips. However, in the presence of propranolol, phenylephrine induced weak detrusor contractions. This result suggests that phenylephrine may activate $\alpha$-1A ARs in both sensory nerves and parasympathetic nerves, and could cause norepinephrine and acetylcholine release form the nerve terminal $[19,20]$. Finally, the effects of $\beta$-ARs overcame those of muscarinic receptors, resulting in detrusor relaxation. Since phenylephrine does not have $\beta$-AR activity, it may indirectly activate adrenergic nerves to release norepinephrine, which does exert $\beta$-AR activity.

We (for the first time, we) demonstrated that inhibition of a-1A ARs may prevent relaxation of the detrusor muscle. Phenylephrine-induced relaxation was inhibited by silodosin, a selective $\alpha-1 \mathrm{~A}$ adrenergic blocker, in UB strips with or without mucosa (Fig. 2). These results suggest that the relaxation effect of phenylephrine occurs through neuronal modification via a-1A AR activity. The $\alpha-1$ ARs mediate prejunctional facilitation to release acetylcholine $[19,20]$ or norepinephrine [19] from nerve fibers. Three subtypes of $\alpha-1$ ARs are present in peripheral tissues: $\alpha-1 \mathrm{~A}, \alpha-1 \mathrm{~B}$, and $\alpha-1 \mathrm{D}$ [13]. These subtypes are expressed in different locations of the UB. $\alpha-1 D$ ARs are expressed in the detrusor muscle in rats with hypertension, bladder outlet obstruction, and advanced age [21], but the pattern of expression is unclear in mice, and $\alpha-1 B$ ARs may be not expressed in the detrusor muscle $[22,23]$. These different $\alpha-1$ ARs mediate relaxation of the detrusor in response to phenylephrine and norepinephrine. Nonselective $\alpha-1$ adrenergic agonists, such as pseudoephedrine and phenylephrine, may induce relaxation of the bladder dome and contraction of the bladder neck and prostate, thereby increasing the risk of urinary retention [24]. Nonselective $\alpha-1$ adrenergic blockers may inhibit detrusor con- 


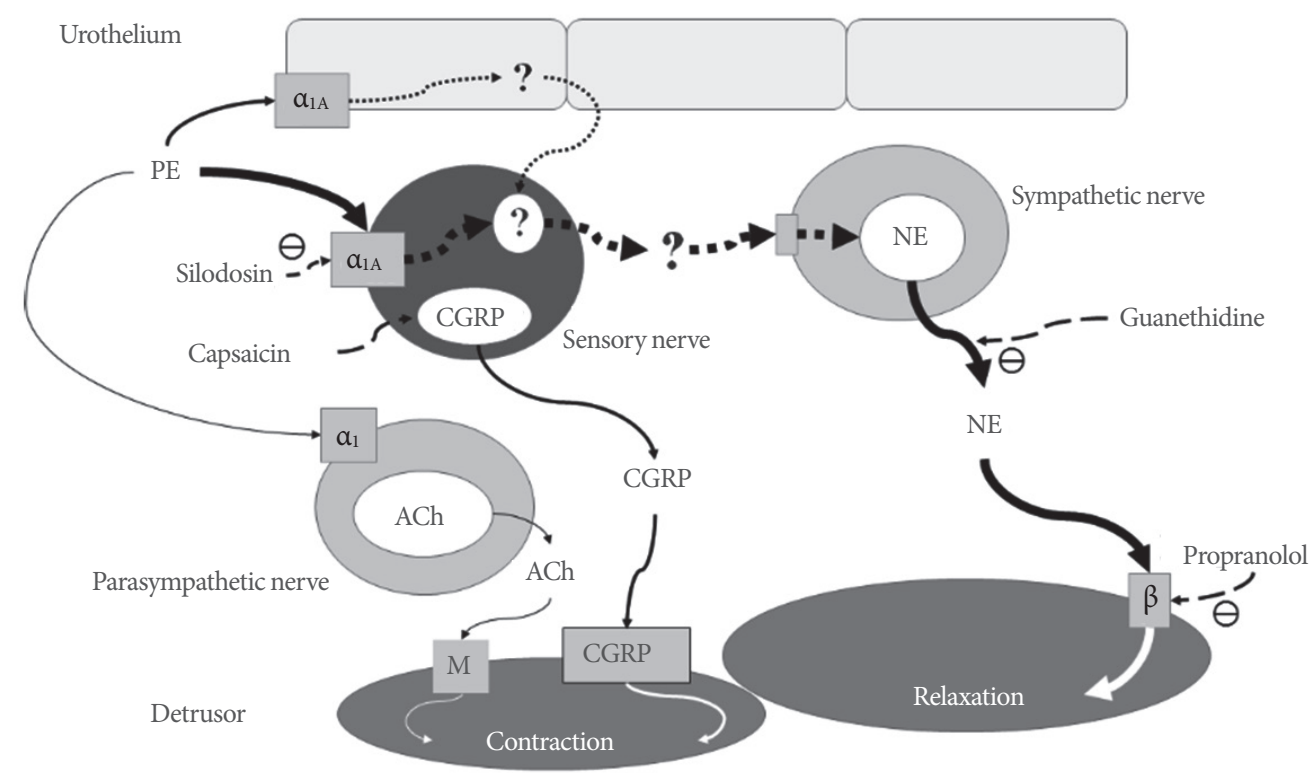

Fig. 6. Schematic illustration of how phenylephrine (PE) induces neurogenic relaxation in the urinary bladder of mice. PE may activate a-1A adrenergic receptors (ARs) of sensory nerves, and then activate capsaicin-sensitive sensory nerves, causing them to release an unknown substance that facilitates the release of norepinephrine inside adrenergic nerves. Subsequently, norepinephrine stimulates $\beta$-ARs in the detrusor muscle, leading to relaxation of the urinary bladder. CGRP, calcitonin gene-related peptide; NE, norepinephrine; ACh, acetylcholine; M, Muscarinic acetylcholine receptor.

traction, which may hamper bladder emptying in cases of detrusor underactivity, while selective $\alpha-1 \mathrm{~A}$ adrenergic blockers may inhibit detrusor relaxation and could ameliorate bladder contraction at voiding. Further clinical studies are required to conclusively determine whether $\alpha-1 \mathrm{~A}$ adrenergic blockers may be a better choice for men with bladder outlet obstruction and detrusor underactivity.

Fig. 6 summarizes the results of the present study. Phenylephrine may activate the $\alpha-1$ A ARs of sensory nerves, and then activate capsaicin-sensitive sensory nerves, causing them to release some unknown substance to facilitate the release of norepinephrine inside adrenergic nerves. Subsequently, norepinephrine stimulates $\beta$-ARs in the detrusor muscle, leading to relaxation of the UB. Depletion of CGRPs by capsaicin and $\alpha-1 \mathrm{~A}$ adrenergic blockers prevented detrusor relaxation induced by phenylephrine.

This study has several limitations. The number of subjects was small, and all findings were in vitro, which may weaken the results presented herein. Further studies with a larger number of subjects and both in vitro and in vivo experiments are required to prove the mechanism proposed in the current study. The current data showing that depletion of CGRPs by capsaicin and $\alpha-1 \mathrm{~A}$ adrenergic blockers prevented phenylephrine-in- duced detrusor relaxation provide indirect evidence suggesting a possible mechanism of detrusor relaxation involving $\alpha-1 \mathrm{~A}$ ARs. Direct immunohistochemical staining of the detrusor muscle should be used to identify CGRP/capsaicin receptors. More studies of the direct effects of various $\alpha-1 \mathrm{~A}$ adrenergic blockers on detrusor function are required to prove or disprove the mechanism we have proposed.

In conclusion, this study demonstrated that sensory nerves might indirectly modulate detrusor motor function though a-1A ARs. Further animal and human studies are required to prove this concept and to validate its clinical usefulness.

\section{AUTHOR CONTRIBUTION STATEMENT}

- Full access to all the data in the study and takes responsibility for the integrity of the data and the accuracy of the data analysis: HH Chang, CK Hsu

- Study concept and design: HH Chang, SJ Chang

- Acquisition of data: HH Chang

- Analysis and interpretation of data: HH Chang, CK Hsu

- Drafting of the manuscript: HH Chang, SJ Chang

- Critical revision of the manuscript for important intellectual content: CH Hsieh, SSD Yang 
- Statistical analysis: HH Chang

- Obtained funding: SSD Yang

- Administrative, technical, or material support: HH Chang, CK $\mathrm{Hsu}$

- Study supervision: $\mathrm{CH}$ Hsieh, SSD Yang

\section{REFERENCES}

1. Awad SA, Bruce AW, Carro-Ciampi G, Downie JW, Lin M, Marks GS. Distribution of alpha- and beta-adrenoceptors in human urinary bladder. Br J Pharmacol 1974;50:525-9.

2. Dunlop D, Shanks RG. Selective blockade of adrenoceptive beta receptors in the heart. Br J Pharmacol Chemother 1968;32:201-18.

3. Levy B, Ahlquist RP. An analysis of adrenergic blocking activity. J Pharmacol Exp Ther 1961;133:202-10.

4. Rivera L, Benedito S, Prieto D, Hernandez M, Labadia A, GarciaSacristan A. Alpha- and beta-adrenoceptors in the sheep urinary bladder. Res Vet Sci 1991;50:259-63.

5. Ueda S, Satake N, Shibata S. Alpha 1- and alpha 2-adrenoceptors in the smooth muscle of isolated rabbit urinary bladder and urethra. Eur J Pharmacol 1984;103:249-54.

6. Meini S, Maggi CA. Evidence for a capsaicin-sensitive, tachykininmediated, component in the NANC contraction of the rat urinary bladder to nerve stimulation. Br J Pharmacol 1994;112:1123-31.

7. Lecci A, Giuliani S, Tramontana M, Santicioli P, Criscuoli M, Dion $S$, et al. Bladder distension and activation of the efferent function of sensory fibres: similarities with the effect of capsaicin. Br J Pharmacol 1998;124:259-66.

8. Giuliani S, Santicioli P, Lippi A, Lecci A, Tramontana M, Maggi $\mathrm{CA}$. The role of sensory neuropeptides in motor innervation of the hamster isolated urinary bladder. Naunyn Schmiedebergs Arch Pharmacol 2001;364:242-8.

9. Trevisani M, Campi B, Gatti R, André E, Materazzi S, Nicoletti P, et al. The influence of alpha1-adrenoreceptors on neuropeptide release from primary sensory neurons of the lower urinary tract. Eur Urol 2007;52:901-8.

10. Aizawa N, Sugiyama R, Ichihara K, Fujimura T, Fukuhara H, Homma Y, et al. Functional roles of bladder al-adrenoceptors in the activation of single-unit primary bladder afferent activity in rats. BJU Int 2016;117:993-1001.

11. Avelino A, Cruz F. Peptide immunoreactivity and ultrastructure of rat urinary bladder nerve fibers after topical desensitization by cap- saicin or resiniferatoxin. Auton Neurosci 2000;86:37-46.

12. Hieble JP, Bondinell WE, Ruffolo RR Jr. Alpha- and beta-adrenoceptors: from the gene to the clinic. 1. Molecular biology and adrenoceptor subclassification. J Med Chem 1995;38:3415-44.

13. Merrill L, Gonzalez EJ, Girard BM, Vizzard MA. Receptors, channels, and signalling in the urothelial sensory system in the bladder. Nat Rev Urol 2016;13:193-204.

14. Ishihama H, Momota Y, Yanase H, Wang X, de Groat WC, Kawatani M. Activation of alpha1D adrenergic receptors in the rat urothelium facilitates the micturition reflex. J Urol 2006;175:35864.

15. Yanase H, Wang X, Momota Y, Nimura T, Kawatani M. The involvement of urothelial alpha1A adrenergic receptor in controlling the micturition reflex. Biomed Res 2008;29:239-44.

16. Birder LA, Nakamura Y, Kiss S, Nealen ML, Barrick S, Kanai AJ, et al. Altered urinary bladder function in mice lacking the vanilloid receptor TRPV1. Nat Neurosci 2002;5:856-60.

17. Sadananda P, Shang F, Liu L, Mansfield KJ, Burcher E. Release of ATP from rat urinary bladder mucosa: role of acid, vanilloids and stretch. Br J Pharmacol 2009;158:1655-62.

18. Aizawa N, Wyndaele JJ. Effects of phenazopyridine on rat bladder primary afferent activity, and comparison with lidocaine and acetaminophen. Neurourol Urodyn 2010;29:1445-50.

19. Somogyi GT, Tanowitz M, de Groat WC. Prejunctional facilitatory alpha 1-adrenoceptors in the rat urinary bladder. Br J Pharmacol 1995;114:1710-6.

20. Yamamoto T, Ghosh R, De Groat WC, Somogyi GT. Facilitation of transmitter release in the urinary bladders of neonatal and adult rats via alpha1-adrenoceptors. Eur J Pharmacol 2001;414:31-5.

21. Ishizuka O, Imamura T, Kurizaki Y, Nishizawa O, Andersson KE. Male lower urinary tract symptoms and a1D-adrenoceptors. Int J Urol 2013;20:73-8.

22. Okutsu H, Matsumoto S, Hanai T, Noguchi Y, Fujiyasu N, Ohtake A, et al. Effects of tamsulosin on bladder blood flow and bladder function in rats with bladder outlet obstruction. Urology 2010;75:23540.

23. Price D. Potential mechanisms of action of superselective alpha(1)adrenoceptor antagonists. Eur Urol 2001;40 Suppl 4:5-11.

24. Shao IH, Wu CC, Tseng HJ, Lee TJ, Lin YH, Tam YY. Voiding dysfunction in patients with nasal congestion treated with pseudoephedrine: a prospective study. Drug Des Devel Ther 2016;10: 2333-9. 